\title{
Nonequilibrium conductance through a benzene molecule in the Kondo regime
}

\author{
L. Tosi ${ }^{1}$ P. Roura-Bas, ${ }^{2}$ and A. A. Aligia ${ }^{1}$ \\ ${ }^{1}$ Centro Atómico Bariloche and Instituto Balseiro, \\ Comisión Nacional de Energía Atómica, 8400 Bariloche, Argentina \\ ${ }^{2}$ Dpto de Física, Centro Atómico Constituyentes, \\ Comisión Nacional de Energía Atómica, Buenos Aires, Argentina
}

(Dated: July 8, 2018)

\begin{abstract}
Starting from exact eigenstates for a symmetric ring, we derive a low-energy effective generalized Anderson Hamiltonian which contains two spin doublets with opposite momenta and a singlet for the neutral molecule. For benzene, the singlet (doublets) represent the ground state of the neutral (singly charged) molecule. We calculate the non-equilibrium conductance through a benzene molecule, doped with one electron or a hole (i.e. in the Kondo regime), and connected to two conducting leads at different positions. We solve the problem using the Keldysh formalism and the non-crossing approximation (NCA). When the leads are connected in the para position (at 180 degrees), the model is equivalent to the ordinary impurity Anderson model and its known properties are recovered. For other positions, there is a partial destructive interference in the cotunneling processes involving the two doublets and as a consequence, the Kondo temperature and the height and width of the central peak (for bias voltage $V_{b}$ near zero) of the differential conductance $G=d I / d V_{b}$ (where $I$ is the current) are reduced. In addition, two peaks at finite $V_{b}$ appear. We study the position of these peaks, the temperature dependence of $G$ and the spectral densities. Our formalism can also be applied to carbon nanotube quantum dots with intervalley mixing.

PACS numbers: 73.23.-b,73.22.-f, 75.20.Hr
\end{abstract}

\section{INTRODUCTION}

Single molecule electronic devices are being extensively studied because they offer perspectives for further miniaturization of electronic circuits with important potential applications $^{1-5}$, and also due to its intrinsic interest in basic research, for example as realizations of the Kondo effect, which manifests experimentally by an increased conductance at low temperatures ${ }^{6.7}$. In addition, quantum phase transitions involving partially Kondo screened spin-1 molecular states were induced changing externally controlled parameters ${ }^{8-10}$. These experiments could be explained semiquantitatively using extensions of the impurity Anderson model treated with either the numerical renormalization group (NRG) ${ }^{9}-11$ or with the noncrossing approximation (NCA $)^{10-13}$. This approximation allows calculations out of equilibrium and in particular at finite bias voltage.

The conductance through benzene-1,4-dithiol has been measured using a mechanically controllable break junction ${ }^{14}$. More recently, other molecules containing benzene rings or related phenyl groups were studied ${ }^{15,16}$. The states nearer to the Fermi level in benzene are built from the $2 \mathrm{p}$ states of the $\mathrm{C}$ atoms which lie perpendicular to the plane of the molecule (the so called $\pi$ states). Transport through aromatic molecules and in particular through benzene in different geometries has been calculated by several groups ${ }^{17-21}$. Hettler et al. 17 started from an exact calculation of an extended Hubbard model for the $\pi$ states, and included the coupling to the leads perturbatively (for couplings smaller than the temperature), to calculate the current through neutral benzene under an applied bias voltage $V_{b}$ for zero gate voltage $V_{g}$, includ- ing radiative relaxation. Cardamone et $a l .18$, described the molecule by a Hubbard model supplemented by repulsions at larger distance (the so called Pariser-Parr-Pople (PPP) Hamiltonian ${ }^{22}$ ) using the self-consistent HartreeFock approximation. The conductance of the effective one-body problem for small $V_{g}$ has been calculated using the Landauer-Büttiker formalism ${ }^{23}$, The authors propose to exploit the destructive interference and to control the conductance of the device introducing symmetry breaking perturbations. Similar results were obtained using $a b$ initio calculations ${ }^{19}$, which have also the drawback of neglecting the effect of correlations.

This effect together with that interference were included in more recent works which started from the exact solution of the PPP mode ${ }^{20,21}$, as well as in previous works which discussed the conductance through molecules or rings of quantum dots (QDs) threaded by a magnetic flux ${ }^{24-29}$. The conductance $G$ depends on which sites of the molecule are coupled to the leads and on interference phenomena related to the symmetry of the system ${ }^{20,21,29}$. For certain conditions $G$ can be totally suppressed and restored again by symmetrybreaking perturbations ${ }^{21}$. While the main conclusions are safe in general, the coupling to the leads was included in some perturbative approach, either by a Liouville equation method 20 , or using the Jagla-Balseiro formula (JBF) for the conductance ${ }^{24,26}$, which assumes a non-degenerate ground state. These approaches miss the Kondo effect which is non-perturbative in the coupling to the leads 28 . This is particularly important when the gate voltage is such that the benzene molecule becomes charged, because the conductance is larger ${ }^{21}$.

The JBF has also been used to predict that the trans- 
mittance integrated over a finite energy window $24,25,27$ and the equilibrium conductance 25,29 through a ring of strongly correlated one-dimensional systems display dips as a function of the applied magnetic flux at fractional values of the flux quantum, due to destructive interference at crossings of levels with different charge and spin quantum numbers ${ }^{29}$ (as a consequence of spin-charge separation). Recently, we have confirmed the presence of dips in the current under a finite applied bias voltage at low temperatures, using the low-energy effective Hamiltonian, consisting of two doublets and a singlet, for the case of perfect destructive interference ${ }^{30}$. At equilibrium (zero bias voltage), the conductance as a function of temperature for this particular case has been calculated with $\mathrm{NRG}^{31}$. The interplay between interference and interactions were also studied for spinless electrons in multilevel systems ${ }^{32}$, and benzene attached to two leads ${ }^{33}$. Although at first sight they might seem artificial, spinless models describe effective Hamiltonians for realistic systems under applied magnetic fields 34,35 .

Interference phenomena were observed in systems with QDs. In a multilevel QD, the crossing of levels and the ensuing destructive interference has been induced applying magnetic field to a system with large, level-dependent $g$ factors 34 . Aharonov-Bohm oscillations were observed in systems involving two quantum dots (QDs) $)^{36}$.

In this work, we construct the low-energy effective Hamiltonian for a ring of $n$ sites with one orbital per site and symmetry that includes the point group $C_{n v}$ (or $C_{n}$ ), weakly coupled to two conducting leads, retaining for $n$ even, the lowest singlet with $n$ particles and the two lowest doublets with $n+1$ particles (electrons or holes depending on the sign of the applied gate voltage $V_{g}$ ). For $n$ odd, the charge of doublets and singlets are interchanged. Using a gauge transformation, three of the four hopping matrix elements between the doublets and the leads can be made real. The phase of the fourth one $\phi$ is in general different from zero and depends on the position of the leads and the wave vectors of the states involved. While the coupling to the leads should be small in such a way that the neglected states do not affect the calculations $^{37}$, we treat this coupling in a self-consistent, non perturbative way, using the the Keldysh formalism of the NCA for problems out of equilibrium $13,38,39$, appropriately extended for this problem. We calculate the non-equilibrium conductance for case of benzene $(n=6)$ in a regime of $V_{g}$ for which the doublets are favored, leading to Kondo effect.

For the case of one doublet, comparison of NCA with NRG results ${ }^{40}$, shows that the NCA describes accurately the Kondo physics. The leading behavior of the differential conductance for small voltage and temperature 41 agrees with alternative Fermi-liquid approaches ${ }^{42,43}$, and the temperature dependence of the conductance practically coincides with the NRG result over several decades of temperature $\stackrel{41}{2}$. A shortcoming of the $\mathrm{NCA}$ is that, at very low temperatures, it introduces an artificial spike at the Fermi energy in the spectral density when the ground state of the system without coupling to the leads (zero hybridization) is non-degenerate, although the thermodynamic properties continue to be well described ${ }^{38}$. This is not important in the present work, where the doublets are below the singlet and there is no applied magnetic field. Another limitation of the method is that it is restricted to temperatures above $\sim T_{K} / 20$, where $T_{K}$ is the Kondo temperature. In our case, this limitation is not important either because as we shall see, the conductance has already saturated at temperatures above this limit. A virtue of the NCA that becomes important in our case, is its ability to capture features at high energies, such as peaks in the spectral density out of the Fermi level, which might be lost in NRG calculations 44 . An example is the plateau at intermediate temperatures observed in transport through $\mathrm{C}_{60}$ molecules for gate voltages for which triplet states are important ${ }^{8,10}$, which was missed in early NRG studies, but captured by the $\mathrm{NCA}^{12,13}$. More recent NRG calculations using tricks to improve the resolution ${ }^{45}$, have confirmed this plateau ${ }^{10}$.

For the particular case of perfect destructive interference $\phi=\pi$ (which does not correspond to benzene), the spectral densities 46 and the non-equilibrium current ${ }^{30}$, were calculated before for symmetric coupling to the leads. In this case, the model is equivalent to an SU(4) impurity Anderson model, used by several authors $47-50$ to interpret transport experiments in $\mathrm{C}$ nanotubes ${ }^{51,52}$, and more recently in Si fin-type field effect transistors ${ }^{53}$. If in addition a finite splitting $\delta$ between the doublets is allowed, the symmetry is reduced to $\mathrm{SU}(2)$. The transition between $\mathrm{SU}(4)$ and $\mathrm{SU}(2)$ has been studied by several authors using the $\mathrm{NCA}^{30,46,49,53}$. In particular we found that the reduction of the Kondo temperature with $\delta$ agrees very well with a simple formula obtained from a variational calculation 30,46 . An important difference between our model and those used for other systems is the connectivity with the leads, which imposes that in our case, the equilibrium conductance $G\left(V_{b}=0\right)$ is zero in the $\mathrm{SU}(4)$ limit $\delta=0$, and therefore, it is not proportional to the total spectral density of $\operatorname{states}^{30}$. For $\phi=\pi$ and any $\delta$, it is possible to calculate $G(0)$ in terms of static occupations, using a generalized Friedel-Langreth sum rule ${ }^{30}$, due to the simplicity of the "orbital" field $\delta$, which acts in the same way as a magnetic field, and allows to relate the phase shift for each channel and spin with the respective occupancies. In turn, these phase shifts enter a general Fermi-liquid expression for the conductance obtained by Pustilnik and Glazman 54 . This is not possible for general $\phi$. Therefore a novel treatment is needed to calculate the conductance even at equilibrium.

Recently, it has been shown that in carbon nanotube QDs with disorder induced valley mixing, the SU(4) symmetry is broken and the tunnel couplings to metallic leads become complex and depend on the applied magnetic field ${ }^{55}$. The present formalism is also suitable to study this case.

The paper is organized as follows. In Section II we describe the construction of the effective Hamiltonian. The 


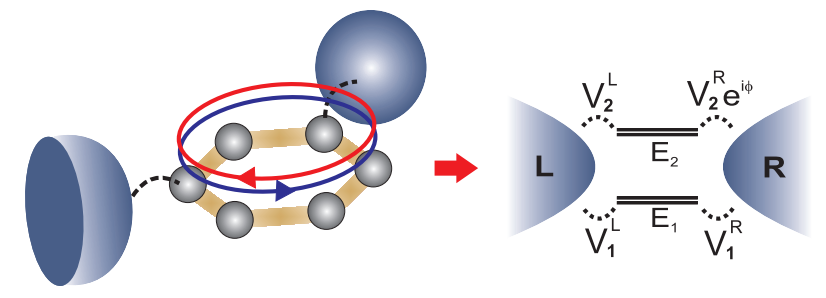

FIG. 1. (Color online) Scheme of the relevant matrix elements at low energies.

approximations and the equation for the current are presented in Section III Section IV contains the numerical results for transport through the benzene molecule and their interpretation. Section $\nabla$ contains a summary and a short discussion. In appendix $\mathrm{A}$ we show that the $\mathrm{NCA}$ conserves the current, extending the existing demonstration for the case of one $\operatorname{doublet}^{38}$. Appendix $\mathrm{B}$ contains some numerical details.

\section{MODEL}

The effective model, which is represented at the right of Fig. 11 contains a singlet with total wave vector $K_{0}$ (usually 0 or $\pi$ ) and two doublets with wave vectors $K_{1}$ and $K_{2}$, that represent the states of lowest energy of two neighboring configurations of a ring of $n$ sites with symmetry $C_{n v}$. In the case of benzene, they correspond to the singlet ground state, invariant under rotations $\left(K_{0}=0\right)$ and two degenerate doublets with total wave vectors $\pm K$, which are the ground state of the molecule for one added electron or hole, depending on the sign of the applied gate voltage $20,21,29$. For one added hole $K=\pi / 3$, while for one added electron $K=2 \pi / 3$. The effective Hamiltonian is

$$
\begin{aligned}
H= & E_{s}|s\rangle\left\langle s\left|+\sum_{i \sigma} E_{i}\right| i \sigma\right\rangle\langle i \sigma|+\sum_{\nu k \sigma} \epsilon_{\nu k} c_{\nu k \sigma}^{\dagger} c_{\nu k \sigma} \\
& +\sum_{i \nu k \sigma}\left(V_{i}^{\nu}|i \sigma\rangle\langle s| c_{\nu k \sigma}+\text { H.c. }\right)
\end{aligned}
$$

where the singlet $|s\rangle$ and the two doublets $|i \sigma\rangle(i=1,2$; $\sigma=\uparrow$ or $\downarrow)$ denote the localized states, $c_{\nu k \sigma}^{\dagger}$ create conduction states in the left $(\nu=L)$ or right $(\nu=R)$ lead, and $V_{i}^{\nu}$ describe the hopping elements between the leads and both doublets, assumed independent of $k$. This hopping element is calculated $\mathrm{as}^{29}$

$$
V_{i}^{\nu}=t_{\nu}\left\langle i \sigma\left|c_{j_{\nu} \sigma}^{\dagger}\right| s\right\rangle
$$

where $c_{j \sigma}^{\dagger}$ creates an electron (or hole) with spin $\sigma$ at the $\pi$ orbital at site $j$ ( 1 to $n$ ) of the molecule, $j_{\nu}$ denotes the site connected to the lead $\nu$, and $t_{\nu}$ is the hopping between this site and the lead $\nu$.

Choosing adequately the phases of the gauge transformation $|i \sigma\rangle \rightarrow e^{i \varphi_{i}}|i \sigma\rangle$, both $V_{i}^{L}$ can be made real and positive, and by reflection symmetry $V_{1}^{L}=V_{2}^{L}$. Using rotational symmetry, it is easy to see that 29

$$
V_{i}^{R}=\left(t_{R} / t_{L}\right) V_{i}^{L} \exp \left[-i\left(j_{R}-j_{L}\right)\left(K_{i}-K_{0}\right)\right]
$$

where $K_{i}$ is the wave vector of $|i \sigma\rangle$. The phase of $V_{1}^{R}$ can be absorbed by a gauge transformation in the $c_{R k \sigma}$, rendering it real and positive. The remaining matrix element is $V_{2}^{R}=V_{1}^{R} e^{-i \phi}$, where $\phi=\left(j_{R}-j_{L}\right)\left(K_{2}-K_{1}\right)$. This result is general for a ring geometry.

For benzene $K_{2}-K_{1} \equiv \pm 2 \pi / 3$. Then, if the leads are connected in the para position $\left(j_{R}-j_{L}=3\right), \phi \equiv 0$, while in the ortho $\left(j_{R}-j_{L}=1\right)$ or meta $\left(j_{R}-j_{L}=2\right)$ positions, $\phi \equiv \pm 2 \pi / 3$. Note that the sign of $\phi$ does not affect our results. For simplicity we shall assume $\left|t_{R} / t_{L}\right|=1$ in the calculations presented here. Then, the hopping of the leads to the relevant states of the benzene molecule are $V_{1}^{L}=V_{2}^{L}=V_{1}^{R}=V, V_{2}^{R}=V e^{-i \phi}$, with $\phi=0$ for the para position, and $\phi= \pm 2 \pi / 3$ for the other two possibilities of connecting the leads.

For $\phi=0$, the state $|B \sigma\rangle=(|1 \sigma\rangle-|2 \sigma\rangle) / \sqrt{2}$ decouples from the leads and the transport properties of the system are the same as those for a single level $[|A \sigma\rangle=$ $(|1 \sigma\rangle+|2 \sigma\rangle) / \sqrt{2}]$ QD connected to he leads, with hopping $\sqrt{2} V$, which are well known $38,56-59$. Another known limit of the model, which cannot be realized in benzene molecules, but in rings of a number of sites multiple of four $^{21}$ is $\phi=\pi$. In this case, $|A \sigma\rangle(|B \sigma\rangle)$ is coupled only to the left (right) lead and the model is equivalent to an SU(4) Anderson model 30,46 . However, in contrast to the case of $\mathrm{C}$ nanotubes ${ }^{47-50}$ and $\mathrm{Si}$ fin-type field effect transistors 53 , the conductance vanishes identically as a consequence of perfect destructive interference ${ }^{30,31}$. An important technical difference is that in our model, the hybridization matrices of the states $|i \sigma\rangle$ with the left and right leads are not proportional for $\phi \neq 0$. If the degeneracy between the levels can be lifted (for example applying an external flux ${ }^{29}$ ), the symmetry is reduced to $\mathrm{SU}(2)$ and a finite conductance is restored ${ }^{30,31,46}$. In $\mathrm{C}$ nanotubes with disorder induced valley mixing ${ }^{55}$, the effective model becomes very similar to ours, including a finite level splitting and a phase $\phi$, which depends on the applied magnetic field.

We shall use our previous results for the case $\phi=\pi$ and a finite level splitting $\delta=E_{2}-E_{1}$ to help in the analysis of the results presented here for benzene.

\section{THE FORMALISM}

In this section we describe the extension of the noncrossing approximation (NCA) applied before for the Anderson model with infinite on-site repulsion out of equilibrium ${ }^{38,39}$, to our effective Hamiltonian. 


\section{A. Representation of the Hamiltonian with slave particles}

An auxiliary boson $b$, and four auxiliary fermions $f_{i \sigma}$ are introduced, so that the localized states are represented as

$$
|s\rangle=b^{\dagger}|0\rangle,|i \sigma\rangle=f_{i \sigma}^{\dagger}|0\rangle
$$

where $|0\rangle$ is the vacuum. These pseudoparticles should satisfy the constraint

$$
b^{\dagger} b+\sum_{i \sigma} f_{i \sigma}^{\dagger} f_{i \sigma}=1
$$

Introducing it by a Lagrange multiplier, the effective Hamiltonian takes the form

$$
\begin{aligned}
H^{\prime}= & \left(E_{s}+\lambda\right) b^{\dagger} b+\sum_{i \sigma}\left(E_{i}+\lambda\right) f_{i \sigma}^{\dagger} f_{i \sigma}+\sum_{\nu k \sigma} \epsilon_{\nu k} c_{\nu k \sigma}^{\dagger} c_{\nu k \sigma} \\
& +\sum_{k \nu i \sigma}\left(V_{i}^{\nu} f_{i \sigma}^{\dagger} b c_{k \nu \sigma}+\text { H.c. }\right) .
\end{aligned}
$$

The NCA solves a system of self-consistent equations to obtain the Green functions of the pseudoparticles (described below), which is equivalent to sum an infinite series of diagrams (all those without crossings) in the corresponding perturbation series in the last term of $H^{\prime}$, and afterwards project on the physical subspace of the constraint. The main new difficulties compared to the case of one doublet arise as a consequence of the matrix structure of the pseudofermion Green functions and self energies, which are absent in the SU(4) case or in this case with simple $\mathrm{SU}(2)$ symmetry breaking perturbations, as a magnetic 53 or "orbital" 30,31,46 field.

\section{B. Green functions}

The lesser and greater Keldysh Green functions for the psedoparticles for stationary non-equilibrium processes are defined as 60,61

$$
\begin{aligned}
G_{i j, \sigma}^{<}\left(t-t^{\prime}\right) & =+i\left\langle f_{j \sigma}^{\dagger}\left(t^{\prime}\right) f_{i \sigma}(t)\right\rangle, \\
D^{<}\left(t-t^{\prime}\right) & =-i\left\langle b^{\dagger}\left(t^{\prime}\right) b(t)\right\rangle, \\
G_{i j, \sigma}^{>}\left(t-t^{\prime}\right) & =-i\left\langle f_{i \sigma}(t) f_{j \sigma}^{\dagger}\left(t^{\prime}\right)\right\rangle, \\
D^{>}\left(t-t^{\prime}\right) & =-i\left\langle b(t) b^{\dagger}\left(t^{\prime}\right)\right\rangle,
\end{aligned}
$$

the retarded and advanced fermion Green functions are $G_{i j, \sigma}^{r}(t)=\theta(t)\left[G_{i j, \sigma}^{>}(t)+G_{i j, \sigma}^{<}(t)\right], G_{i j, \sigma}^{a}=G_{i j, \sigma}^{r}+G_{i j, \sigma}^{<}-$ $G_{i j, \sigma}^{>}$, and similarly for the boson Green functions replacing $G_{i j, \sigma}$ by $D$.

These Green functions correspond to the interacting (dressed) propagators and are determined selfconsistently within the NCA. Evaluating the corresponding diagrams in second order in the $V_{i}^{\nu}$, and replacing the bare propagators by the dressed ones, the expressions for the lesser self-energies take the form

$$
\begin{aligned}
\Pi^{<}(\omega) & =-\sum_{\nu l m \sigma} \Gamma_{l m}^{\nu} \int \frac{d \omega^{\prime}}{2 \pi}\left(1-f_{\nu}\left(\omega^{\prime}-\omega\right)\right) G_{m l, \sigma}^{<}\left(\omega^{\prime}\right), \\
\Sigma_{l m, \sigma}^{<}(\omega) & =-\sum_{\nu} \Gamma_{l m}^{\nu} \int \frac{d \omega^{\prime}}{2 \pi} f_{\nu}\left(\omega-\omega^{\prime}\right) D^{<}\left(\omega^{\prime}\right),
\end{aligned}
$$

where

$$
\Gamma_{i j}^{\nu}(\omega)=2 \pi \sum_{k} V_{i}^{\nu} \bar{V}_{j}^{\nu} \delta\left(\omega-\epsilon_{\nu k}\right)
$$

assumed independent of $\omega$. Similarly, the greater selfenergies become

$$
\begin{aligned}
\Sigma_{l m, \sigma}^{>}(\omega) & =\sum_{\nu} \Gamma_{l m}^{\nu} \int \frac{d \omega^{\prime}}{2 \pi}\left(1-f_{\nu}\left(\omega-\omega^{\prime}\right)\right) D^{>}\left(\omega^{\prime}\right), \\
\Pi^{>}(\omega) & =\sum_{\nu l m \sigma} \Gamma_{l m}^{\nu} \int \frac{d \omega^{\prime}}{2 \pi} f_{\nu}\left(\omega^{\prime}-\omega\right) G_{m l, \sigma}^{>}\left(\omega^{\prime}\right) .
\end{aligned}
$$

As in the case of one doublet only ${ }^{38}$, in the expressions for the retarded self energies, $\Sigma^{r}(t)=-\theta(t)\left(\Sigma^{<}(t)-\right.$ $\left.\Sigma^{>}(t)\right), \Sigma^{<}$can be neglected in comparison with $\Sigma^{>}$due to the effect of the constraint. Then after Fourier transforming one obtains

$$
\begin{aligned}
\Sigma_{i j, \sigma}^{r}(\omega) & =i \int \frac{d \omega^{\prime}}{2 \pi} \frac{\Sigma_{i j, \sigma}^{>}\left(\omega^{\prime}\right)}{\omega-\omega^{\prime}+i \eta} \\
\Pi^{r}(\omega) & =i \int \frac{d \omega^{\prime}}{2 \pi} \frac{\Pi^{>}\left(\omega^{\prime}\right)}{\omega-\omega^{\prime}+i \eta}
\end{aligned}
$$

where $\eta$ is a positive infinitesimal. The advanced self energies $\Sigma_{i j, \sigma}^{a}$ and $\Pi^{a}$ are obtained changing the sign of $\eta$ in the expressions above.

For the case of SU(4) symmetry or when this symmetry is broken by simple symmetry breaking fields $30,31,46-50,53$ ( $\phi=\pi$ and symmetric coupling to the leads in our effective model), the fermion Green functions and self energies are diagonal in an appropriate basis and the self-consistency problem simplifies considerably. In the general case (including $\phi \equiv \pm 2 \pi / 3$ for benzene) one has to solve a matrix Dyson equation 60,61 which includes not only the Keldysh (or +, - branch index in the Keldysh contour), but also the doublet index $i=1,2$ in the fermion case. For the retarded fermion Green functions and self energies, combining $G_{i j, \sigma}^{r}$ in a $2 \times 2$ matrix $\mathbf{G}^{r}$ and similarly for self energies and unperturbed Green functions $g_{i j, \sigma}^{r}=\delta_{i j}\left(\omega-\tilde{E}_{i}\right)^{-1}$, with $\tilde{E}_{i}=E-i+\lambda$ the Dyson equations take the simple form $\mathbf{G}^{r}=\mathbf{g}^{r}+\mathbf{g}^{r} \boldsymbol{\Sigma}^{r} \mathbf{G}^{r}$. Solving the system for the 
$G_{i j, \sigma}^{r}$ we obtain

$$
\begin{aligned}
& G_{11, \sigma}^{r}=\frac{1}{\mathbb{D}}\left(\omega-\tilde{E}_{2}-\Sigma_{22, \sigma}^{r}\right), \\
& G_{12, \sigma}^{r}=\frac{1}{\mathbb{D}}\left(\Sigma_{12, \sigma}^{r}\right), \\
& G_{21, \sigma}^{r}=\frac{1}{\mathbb{D}}\left(\Sigma_{21, \sigma}^{r}\right), \\
& G_{22, \sigma}^{r}=\frac{1}{\mathbb{D}}\left(\omega-\tilde{E}_{1}-\Sigma_{11, \sigma}^{r}\right),
\end{aligned}
$$

where

$$
\mathbb{D}=\left(\omega-\tilde{E}_{1}-\Sigma_{11, \sigma}^{r}\right)\left(\omega-\tilde{E}_{2}-\Sigma_{22, \sigma}^{r}\right)-\Sigma_{12, \sigma}^{r} \Sigma_{21, \sigma}^{r} .
$$

For the boson, which has no subscripts, one has

$$
D^{r}(\omega)=\frac{1}{\omega-\tilde{E}_{s}-\Pi^{r}} .
$$

The advanced Green functions can be obtained from the retarded ones by the replacement $\eta \rightarrow-\eta$.

The remaining equations that relate the lesser and greater pseudoparticle Green functions with the corresponding self energies are (in compact matrix form for the fermions) $)^{62}$

$$
\begin{gathered}
\mathbf{G}^{\lessgtr}=\mathbf{G}^{r} \boldsymbol{\Sigma}^{\lessgtr} \mathbf{G}^{a}, \\
D^{\lessgtr}=D^{r} \Pi^{\lessgtr} D^{a} .
\end{gathered}
$$

We solve numerically the system of integral equations (8) to (14) for the pseudoparticle Green functions and self energies. The details are given in appendix B

For the calculation of the current, one needs the Green functions of the physical fermions $d_{i \sigma}^{\dagger}=|i \sigma\rangle\langle s|=f_{i \sigma}^{\dagger} b$. These functions, which we identify with the subscript $\mathbf{d}$, are defined in the same way as the pseudofermion ones [see Eqs. (7)], replacing $f_{i \sigma}$ by $d_{i \sigma}$. In terms of the auxiliary-particle Green functions, the lesser and greater physical Green functions take the form ${ }^{38}$

$$
\begin{aligned}
& G_{\mathbf{d} i j, \sigma}^{<}(\omega)=i \int \frac{d \omega^{\prime}}{2 \pi Q} G_{i j, \sigma}^{<}\left(\omega^{\prime}+\omega\right) D^{>}\left(\omega^{\prime}\right), \\
& G_{\mathbf{d} i j, \sigma}^{>}(\omega)=i \int \frac{d \omega^{\prime}}{2 \pi Q} G_{i j, \sigma}^{>}\left(\omega^{\prime}+\omega\right) D^{<}\left(\omega^{\prime}\right),
\end{aligned}
$$

where $Q=\left\langle b^{\dagger} b+\sum_{i \sigma} f_{i \sigma}^{\dagger} f_{i \sigma}\right\rangle$ for a given Lagrange multiplier $\lambda$ (see appendix $\mathrm{B}$ ).

\section{Equation for the current}

Using general formulas for the current through a region with interacting electrons $\frac{64,65}{6}$ and the relation $G^{r}-G^{a}=$ $G^{>}-G^{<}$between Green functions, the current of our effective model for a spin degenerate system (without applied magnetic field) can can be written as

$$
\begin{aligned}
I & = \pm \frac{i e}{h} \int d \omega \operatorname{Tr}\left[\left(\boldsymbol{\Gamma}^{\mathbf{L}} f_{L}(\omega)-\boldsymbol{\Gamma}^{\mathbf{R}} f_{R}(\omega)\right) \mathbf{G}_{\mathbf{d}}^{>}(\omega)\right. \\
& \left.+\left(\left(1-f_{L}(\omega)\right) \boldsymbol{\Gamma}^{\mathbf{L}}-\left(1-f_{R}(\omega)\right) \boldsymbol{\Gamma}^{\mathbf{R}}\right) \mathbf{G}_{\mathbf{d}}^{<}(\omega)\right]
\end{aligned}
$$

where the $+(-)$ sign correspond to the case in which the doublets have one more electron (hole) than the singlet, $f_{\nu}(\omega)=\left[\exp \left[\left(\omega-\mu_{\nu}\right) / k T\right]+1\right]^{-1}$ where $\mu_{\nu}$ is the chemical potential of the lead $\nu$, and $\boldsymbol{\Gamma}^{\nu}, \mathbf{G}_{\mathbf{d}}^{<}$and $\mathbf{G}_{\mathbf{d}}^{>}$are $2 \times$ 2 matrices with matrix elements given by Eqs (9) and (15). In particular, taking the unperturbed density of conduction states per spin $\rho=1 /(2 D)$ where $2 D$ is the band width, and symmetric coupling to the leads, we have

$$
\begin{aligned}
\boldsymbol{\Gamma}^{\mathbf{L}} & =\frac{\pi V^{2}}{D}\left(\begin{array}{ll}
1 & 1 \\
1 & 1
\end{array}\right) \\
\boldsymbol{\Gamma}^{\mathbf{R}} & =\frac{\pi V^{2}}{D}\left(\begin{array}{cc}
1 & e^{i \phi} \\
e^{-i \phi} & 1
\end{array}\right) .
\end{aligned}
$$

Note that unless $\phi=0, \Gamma^{\mathbf{L}}$ is not proportional to $\boldsymbol{\Gamma}^{\mathbf{R}}$. As a consequence, the trick used to relate the conductance at $V_{b}=0$ with the spectral density of states 64,65 cannot be used. We calculate the conductance $G=d I / d V_{b}$ by numerical differentiation of the current even for $V_{b} \rightarrow 0$. The traces appearing in Eq. (16) have the form

$$
\begin{aligned}
\operatorname{Tr}\left(\boldsymbol{\Gamma}^{\mathbf{R}} \mathbf{G}_{\mathbf{d}}^{\lessgtr}\right)= & \frac{\pi V^{2}}{D}\left[G_{11}^{\lessgtr}+G_{22}^{\lessgtr}+\cos (\phi)\left(G_{21}^{\lessgtr}+G_{12}^{\lessgtr}\right)\right. \\
& +i \sin (\phi)\left(G_{21}^{\lessgtr}-G_{12}^{\lessgtr}\right),
\end{aligned}
$$

and similarly for $\operatorname{Tr}\left(\boldsymbol{\Gamma}^{\mathbf{L}} \mathbf{G}_{\mathbf{d}}^{\lessgtr}\right)$ replacing $\phi$ by 0 .

Note that from the definition of the Green functions [see Eq. (77)], one realizes that the complex conjugates of the lesser and greater Green functions satisfy $\bar{G}_{i j}^{\lessgtr}(t)=$ $-G_{j i}^{\lessgtr}(-t)$ and after Fourier transform $\bar{G}_{i j}^{\lessgtr}(\omega)=-G_{j i}^{\lessgtr}(\omega)$. Then, $G_{i i}^{\lessgtr}(\omega)$ and $G_{21}^{\lessgtr}(\omega)+G_{12}^{\lessgtr}(\omega)$ are pure imaginary, while $G_{21}^{\lessgtr}(\omega)-G_{12}^{\lessgtr}(\omega)$ is real.

In appendix A we show that the current from the left lead to the molecule equals that from the molecule to the right lead, so that the current is conserved within the NCA. Other approximations, like perturbation theory in the Coulomb repulsion out of the electron-hole symmetric point do not conserve the current ${ }^{43}$, unless some tricks are used $63,67,68$.

\section{NUMERICAL RESULTS}

For the numerical calculations, we assume a constant density of states per spin of the leads $\rho$ between $-D$ and $D$. We take the unit of energy as the total level width of both doublets: $\Gamma=\Gamma_{i i}^{L}+\Gamma_{i i}^{R}=4 \pi \rho V^{2}, i=$ 1,2. Also $\Gamma=2 \Delta$, where $\Delta$, called the resonance level width, is half the width at half maximum of the spectral density of states of particles for each level in the noninteracting case. We restrict our present study to gate voltages such that $E_{s}-E_{i} \gg \Delta$, for which correlations play a more important role, the Kondo effect develops and the conductance is higher. Without loss of generality, we take $\epsilon_{F}=E_{s}=0$, where $\epsilon_{F}$ is the Fermi level of 
the leads without applied bias voltage. We define $E_{d}=$ $E_{1}, \delta=E_{2}-E_{1}$. For a bias voltage $V_{b}$, the chemical potentials are $\mu_{L}=e V_{b} / 2, \mu_{R}=-e V_{b} / 2$.

In the numerical results an important low-energy scale is the Kondo temperature $T_{K}$. It is known that for impurity Anderson models like ours for $\delta=0$ in the Kondo regime, the spectral density shows one charge-transfer peak at energy $E_{d}$ below the Fermi energy of total width of the order of $N \Gamma$ for $\mathrm{SU}(\mathrm{N})$ models $\$ 6,47,66$. For finite onsite Coulomb repulsion $U$ there is another charge-transfer peak at energy $E_{d}+U$, but this is shifted to infinite energies in our case. In addition, there is a narrow peak near the Fermi energy of width of the order of $T_{K}$. In this work we define $T_{K}$ as the half width at half maximum of this peak. For finite $\delta$ additional peaks appear as shown in Section IVB

\section{A. Conductance out of equilibrium}

In Fig. 2 we show the differential conductance $G=$ $d I / d V_{b}$ as a function of bias voltage $V_{b}$, for the leads connected at 180 degrees (in the para position), for which $\phi=0$, and in the other two positions $(\phi= \pm 2 \pi / 3)$, for several values of the energy of the doublets $E_{1}=E_{2}=$ $E_{d}$. The charge-transfer energy $\epsilon_{F}-E_{d}$ is the energy necessary to take a particle (electron or hole) from the Fermi energy, and add it to the localized singlet, forming a doublet, in absence of the hopping to the leads. It is tuned modifying the gate voltage $V_{g}: E_{d}$ decreases with increasing $V_{g}$. The temperature in the curve was fixed at $T=0.05 T_{K}$. Since we have assumed symmetric coupling to both leads, $G\left(-V_{b}\right)=G\left(V_{b}\right)$ and only positive $V_{b}$ are duisplayed in the figure.

For the para position, as explained in Section 2, the problem is equivalent to the transport through a single level quantum dot, and $G$ shows a single peak centered near zero voltage and width of the order of $2 k T_{K} / e^{69}$. We remind the reader that half the width of $G\left(V_{b}\right)$ for $T=0$ times the electric charge $e$, half the width of the spectral density and the temperature (times the Boltzman constant) $T$ at which $G(T)$ for $V_{b}=0$ falls to half of the maximum value, are all of the same order 69 . This is natural from the expected universal behavior of the (single level) Anderson model in the Kondo regime, in which only one energy scale $T_{K}$ survives. This Kondo temperature varies exponentially with the charge-transfer energy $E_{d}$, and as a consequence, the width of the central peak observed in the figure also decreases exponentially with increasing $\left|E_{d}\right|$, as observed in Fig. 2

When the molecule is attached to the leads at 60 or 120 degrees, three main changes occur in $G\left(V_{b}\right)$ in comparison to the previous case: i) The maximum of $G(0)$ is lower, ii) The width of the central peak is narrower and scales in a different way with $E_{d}$, and iii) two new peaks appear at finite $V_{b}$. i) is due to the effect of partial destructive interference between the current transmitted by the two doublets. In the para position, for which all

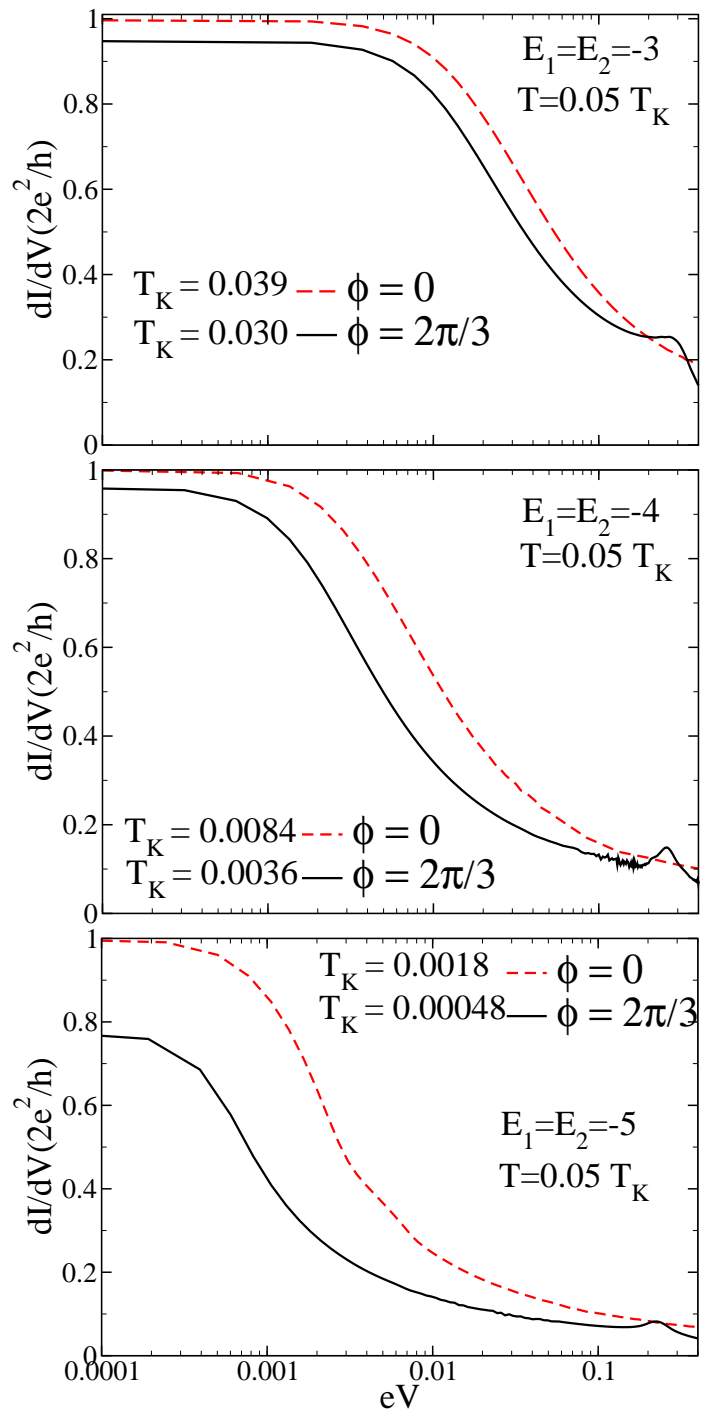

FIG. 2. (Color online) Differential conductance at low temperature through a benzene molecule as a function of bias voltage for several values of the energy of the doublets, with the leads connected in the para (red dashed line) and ortho or meta (full black line) positions.

hybridization to the leads have the same sign $(\phi=0)$, $G(0)$ equals the quantum of conductance $2 e^{2} / h$ for symmetric coupling to the leads (as we have assumed in the present calculations). In the opposite case (hypothetical for benzene) of $\phi=\pi$, there is perfect destructive interference and $G$ vanishes ${ }^{30,31}$. Transport through the benzene molecule connected in the ortho or meta positions, correspond to an intermediate situation $(\phi= \pm 2 \pi / 3)$, and therefore, an intermediate value of $G(0)$ is expected. For asymmetric coupling to the leads, as it is usually the case in transport through molecules in devices built by electromigration ${ }^{8,10}, G(0)$ decreases strongly and it is not possible to distinguish between different positions of the leads connected to the benzene molecule from the maximum value of $G$. Note that this value decreases slightly 


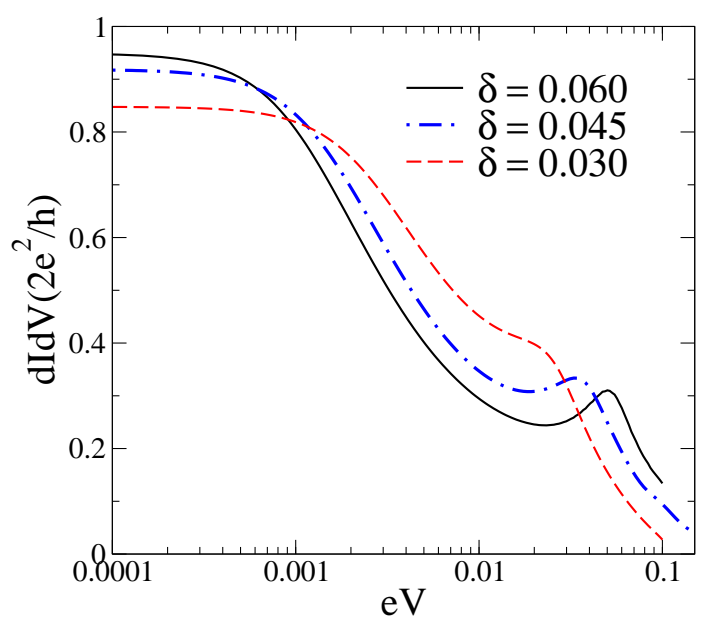

FIG. 3. (Color online) Differential conductance for $\phi=\pi$ and different values of the level splitting. Other parameters are $E_{d}=-4, T=0.05 T_{K}$.

with decreasing $T_{K}$. The feature, which is probably the most useful to distinguish experimentally among the different positions of the conducting leads is the presence of the side peaks in $G\left(V_{b}\right)$, which seem to depend weakly on the charge transfer energy $\epsilon_{F}-E_{d}$, in contrast to the width of the central peak.

In order to help in the interpretation of the results, we calculate $G\left(V_{b}\right)$ for $\phi=\pi$, but allowing for a finite splitting $\delta=E_{2}-E_{1}$ between both doublets. $\delta$ acts as a symmetry breaking field on the $\mathrm{SU}(4)$ Kondo effect $\mathrm{Bu}^{30,46}$. The result is shown in Fig. 3. Clearly, $G\left(V_{b}\right)$ is qualitatively similar to the corresponding result for benzene with leads connected in the ortho or meta positions. The conductance, which vanishes in the $\mathrm{SU}(4)$ limit $\delta=0$ is restored by a finite $\delta$ and two peaks at $e V_{b}= \pm \delta$ appear. This similarity suggest to interpret the results for benzene starting from those for $\phi=\pi, \delta=0$ and thinking the difference between the coupling $V_{2}^{R}$ for $\phi= \pm 2 \pi / 3$ and $\phi=\pi$ as a perturbation. This perturbation, in second order, introduces among other effects, an effective mixing between the levels, proportional to $V^{2} /\left|E_{d}\right|$, which leads to a splitting of the doublets. In fact, the position of the satellite peaks in Fig. 2 (ranging from 0.3 for $E_{d}=-3$ to 0.22 for $E_{d}=-5$ ) is roughly consistent with a $1 /\left|E_{d}\right|$ dependence.

Note that in the case $\phi=0$ in which this perturbation is expected to be the largest, only the symmetric combinations of left and right lead states hybridize with the impurity, while the antisymmetric ones remain decoupled. The spectral density of the latter is a delta function $\delta\left(\omega-E_{d}\right)$, without weight near the Fermi energy. This makes clear that actually the effective hopping of the two resulting split doublets is different and the proposed interpretation is rather qualitative.

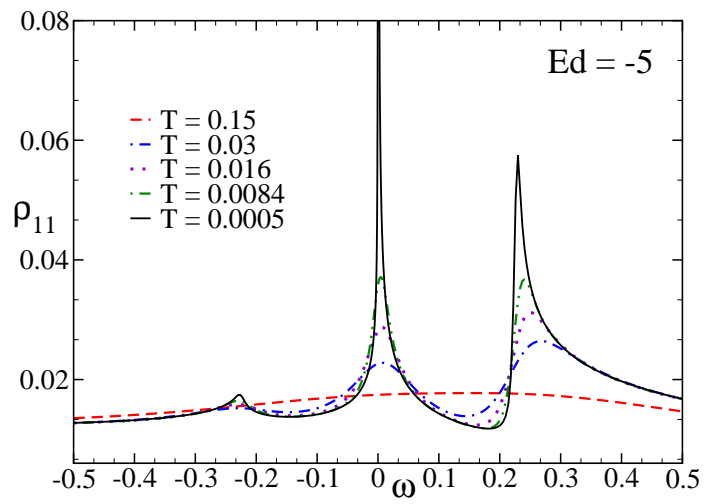

FIG. 4. (Color online) Equilibrium spectral density for the benzene doublets as a function of frequency in the ortho and meta positions for different temperatures.

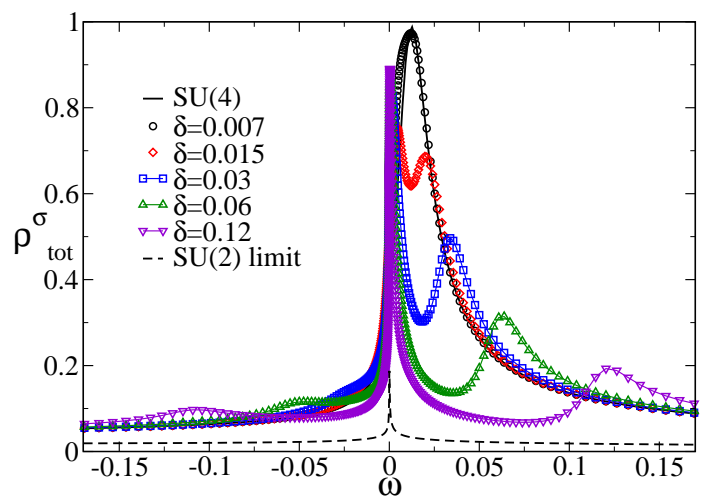

FIG. 5. (Color online) Total spectral density at equilibrium as a function of frequency for the effective model with $\phi=\pi$, $T=0.05 T_{K}$ and different values of the level splitting.

\section{B. Spectral densities}

As a further test of the interpretation outlined above, we compare the spectral density of states of both models. The spectral density

$$
\rho_{i \sigma}(\omega)=\left(G_{\mathbf{d} i i \sigma}(\omega+i \eta)-G_{\mathbf{d} i i \sigma}(\omega-i \eta)\right) /(2 \pi i)
$$

of both doublets for benzene connected to the leads in ortho or meta positions is represented in Fig. 4 for different temperatures and energies near the Fermi energy (the charge-transfer peak ${ }^{46}$ is not shown). As $T$ decreases below $T_{K}$, not only a peak develops near the Fermi energy, but also two side peaks (the most prominent for positive frequencies) are clearly present. In Fig. 5 we show the total spectral density for $\phi=\pi$, very low temperatures and different values of $\delta$. In this case, the peak near to the Fermi energy corresponds to the lowest doublet and the peak at energy near $\delta$ corresponds to the highest doublet $\underline{46}$. In contrast, each peak in Fig. 4 is expected to come from some linear combination of both doublets which is not easy to identify. Another difference apparent in the figure is that the side peak at positive frequencies for benzene is sharper and more asymmetric 


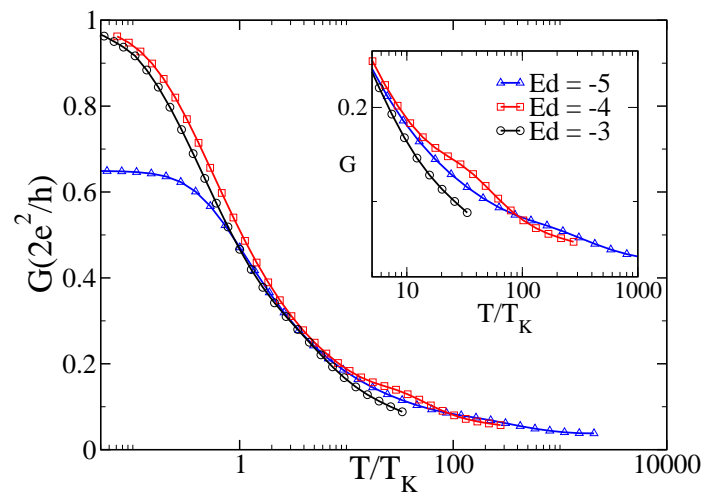

FIG. 6. (Color online) Conductance as a function of temperature for leads connected in the ortho or meta position and several values of the energy of the doublets.

than the corresponding one for $\phi=\pi$ and finite $\delta$. This might be due in part to a smaller effective hybridization of the excited doublet in the case of benzene, leading to a narrower peak, as in the limit $\phi \rightarrow 0$ discussed above.

In spite of these differences, the spectral density for $\phi=\pi$ and finite $\delta$ shows the development of a satellite peak departing from the central one as $\delta$ increases. The spectral density for benzene shows the same qualitative features (a peak near the Fermi energy and another one at positive frequencies) which can be interpreted, in a qualitative first approximation, as coming from an effective $\delta$. This peak at finite energy in turn gives rise to the side peaks in $G\left(V_{b}\right)$ (although the spectral densities are modified with the applied bias voltage and the peaks are blurred).

\section{Temperature dependence of the conductance}

In Fig. 6, we show the evolution with temperature of the conductance $G(T)$ for $V_{b}=0$, several values of $E_{d}$, and conducting leads in the ortho or meta position. $G(T)$ was obtained differentiating the current, as explained in Section IIC In order to be able to display all curves in the same scale, a logarithmic scale of relative temperatures $T / T_{K}$ is used, where the characteristic energy scale $T_{K}$ is given by the half width at half maximum of the equilibrium spectral density $\rho_{i \sigma}(\omega)$. The resulting value of $T_{K}$ is $3 \times 10^{-2}, 3.6 \times 10^{-3}$ and $4.8 \times 10^{-4}$ for $E_{d}=-3$, -4 and -5 respectively. The peak at finite energy in the spectral density at low temperatures (which lies between 0.3 for $E_{d}=-3$ to 0.22 for $E_{d}=-5$ ) results in a structure in $G(T)$ at $T / T_{K}$ near 10,70 and 400 for $E_{d}=-3$, -4 and -5 respectively. While for $E_{d}=-3$ this structure is hidden in the main peak, a kind of plateau is evident for the other values of $E_{d}$ at the corresponding positions, as shown in the inset of Fig. [6.

This structure is reminiscent of the plateau observed by transport experiments in the "triplet" side of the quantum phase transition in $\mathrm{C}_{60} \frac{8,10}{}$, and explained by $\mathrm{NCA}^{10,12,13}$ and NRG calculations with improved resolution 10 , In our case, to represent accurately the high energy features, it was necessary to use a special mesh in the frequency axis with more points near the high energy peak in the pseudofermion spectral densities (see appendix (B).

In previous work ${ }^{30.46}$, we have found that for $\phi=\pi$ and any level splitting $\delta=E_{2}-E_{1}, T_{K}$ (except for a constant of order unity) is very accurately given by the expression

$$
T_{K}=\left\{(D+\delta) D \exp \left[\pi E_{1} /(2 \Delta)\right]+\delta^{2} / 4\right\}^{1 / 2}-\delta / 2,
$$

obtained from a simple variational wave function that generalizes the proposal of Varma and Yafet $\underline{70}$ for the simplest impurity Anderson model. This equation interpolates between the $\mathrm{SU}(4)$ limit $\delta=0$, for which $T_{K}$ has the largest value $T_{K}^{4}=D \exp \left[\pi E_{1} /(4 \Delta)\right]$ and the onelevel SU(2) limit $\delta \rightarrow+\infty$. The effect of $\delta$ on $T_{K}$ is small when $\delta<T_{K}^{4}$, while for larger $\delta, T_{K}$ decreases strongly. Note that $T_{K}^{4}$ coincides with the Kondo temperature of the equivalent one-level effective Anderson for benzene connected in the para position (because the effective onelevel resonant level width contains a factor 2, see Section III). Therefore, we can interpret the fact that the effective energy scale in the ortho and meta positions is smaller than that in the para positions (compare the widths of the central peaks in Fig. 22) as an effect of the effective level splitting caused by a phase $\phi$ different from $\pi$.

In other words, it seems that the position of the side peaks in $G\left(V_{b}\right)$ (or spectral densities) and the plateau in $G(T)$ indicate an effective $\delta$, which decreases the characteristic energy scale $T_{K}$. While we do not expect Eq. (20) to describe accurately $T_{K}$ using this effective $\delta$, the fact that the difference between the energy scales for the para and the other positions is more noticeable for $\delta$ larger than $T_{K}^{4}$ ( $T_{K}$ for the para case), suggests that this equation might be useful for a qualitative understanding, and supports the interpretation of our results.

\section{SUMMARY AND DISCUSSION}

We have constructed the effective Hamiltonian for transport through a symmetric ring (point group including $C_{n v}$ as a subgroup) with one orbital per site, including a singlet and two degenerate doublets of a neighboring configuration for the isolated ring. This includes for example the ground state of the isolated ring for one electron per site, and another with one added electron or hole. The resulting effective generalized Anderson Hamiltonian describes however more general situations. An extension to the case in which a magnetic flux is added (reducing the symmetry to $C_{n}$ and breaking the doublet degeneracy) is trivial. Partial destructive interference occurs in general when two levels with $N$ particles are near to another one with $N \pm 1$ particles 29 . Therefore, the situation of one singlet and two doublets appears frequently. 
From the wave vectors of these states, it is easy to calculate the phase $\phi$ of the effective model following the lines of Section II. Therefore, we expect that our formalism can be used in a variety of physically relevant systems.

We have used the resulting effective Hamiltonian to calculate the non-equilibrium transport through a benzene molecule with conducting leads connected in different positions, using an appropriate generalization of the NCA to include the Kondo effect as well as effects of partial destructive interference between the transport channels through both doublets. While for leads connected in the para position, the conductance $G$ as a function of voltage and temperature is similar to that well known for the case of only one doublet, in the other positions the peak in $G\left(V_{b}\right)$ near zero bias voltage $V_{b}$ is narrower and of smaller intensity as an indication of the relative position of the conducting leads. In addition, $G\left(V_{b}\right)$ displays two additional side peaks at finite $\pm V_{b}$, and a characteristic plateau is present in the conductance as a function of temperature $G(T)$. These finite energy features are probably easier to detect experimentally as an indication of the relative position of the conducting leads.

These results for the leads connected in the ortho and meta positions, which are due to partial destructive interference, can be interpreted starting from those with total destructive interference (corresponding to a phase $\phi=\pi$ ) for which the effective model has SU(4) symmetry, and adding a symmetry breaking splitting $\delta$ between effective doublets caused by the remaining term treated as a perturbation.

For our results to be valid, the coupling to the leads should be sufficiently small, so that excited states, not included in the effective Hamiltonian, do not play an important role. The effect of these states can be estimated for each particular case. See for example Ref ${ }^{37}$. The magnitude of the coupling can be controlled for example in break junctions $\mathbf{s}^{6,9,14}$.

Our effective model, including a finite level splitting $\delta$ from the beginning, and a general phase $\phi$ describes carbon nanotube QDs with disorder induced valley mixing 55 . Therefore, our formalism supplemented by realistic calculations of $\delta$ and $\phi$ might be used to calculate the conductance of these systems.

In this work, we have neglected the effect of phonons, which can modify the effective parameters of the model ${ }^{72,73}$, and also affect the interference phenomena ${ }^{74}$. We have also neglected electrostatic interactions between the leads and the molecules. In the weak coupling regime, it has been shown that interaction with image charges for the doped molecules leads to a symmetry breaking and a splitting of the degenerate levels that also affects the interference phenomena ${ }^{75}$. As we have shown, in the specific case of benzene, the coupling to the leads already originates a splitting of this kind. Therefore, we expect that these electrostatic effects are more relevant in the case of perfect destructive interference.

\section{ACKNOWLEDGMENTS}

We thank CONICET from Argentina for financial support. This work was partially supported by PIP 11220080101821 of CONICET and PICT R1776 of the ANPCyT, Argentina.

\section{Appendix A: Current conservation}

Here we present a brief demonstration that within NCA, the current established between the left metallic contact and the central region (ring) $I_{L}$, is the same as the current flowing from this region to the right metallic contact $I_{R}$. For simplicity we assume that there is no applied magnetic field. The demonstration can be easily extended to the case of inequivalent spins. Adding both spins the expressions of the currents are $\underline{\underline{64}}$

$$
\begin{aligned}
I_{L} & = \pm \frac{2 i e}{h} \int d \omega \operatorname{Tr}\left\{\boldsymbol { \Gamma } ^ { \mathbf { L } } \left[f_{L}(\omega) \mathbf{G}_{\mathbf{d}}^{>}(\omega)\right.\right. \\
& \left.\left.+\left(1-f_{L}(\omega)\right) \mathbf{G}_{\mathbf{d}}^{<}(\omega)\right]\right\}, \\
I_{R} & = \pm \frac{-2 i e}{h} \int d \omega \operatorname{Tr}\left\{\boldsymbol { \Gamma } ^ { \mathbf { R } } \left[f_{R}(\omega) \mathbf{G}_{\mathbf{d}}^{>}(\omega)\right.\right. \\
& \left.\left.+\left(1-f_{R}(\omega)\right) \mathbf{G}_{\mathbf{d}}^{<}(\omega)\right]\right\},
\end{aligned}
$$

where the symbol \pm and the meaning of the matrices is explained in Section III [actually Eq. (16) is the average of Eqs. (A1) and (A2)].

We use Eqs. (15) to replace the physical Green functions by their expressions in terms of the Green functions for the auxiliary particles. Denoting $\mathbf{G}$ the $2 \times 2$ matrix with the pseudo fermion Green functions for a given spin,. one obtains

$$
\begin{aligned}
I_{L} & =\mp \frac{2 e}{h} \int d \omega \int \frac{d \omega^{\prime}}{2 \pi Q} f_{L}(\omega) D^{<}\left(\omega^{\prime}\right) \operatorname{Tr}\left[\Gamma^{\mathbf{L}} \mathbf{G}^{>}\left(\omega^{\prime}+\omega\right)\right] \\
& +\left(1-f_{L}(\omega)\right) D^{>}\left(\omega^{\prime}\right) \operatorname{Tr}\left[\boldsymbol{\Gamma}^{\mathbf{L}} \mathbf{G}^{<}\left(\omega^{\prime}+\omega\right)\right] \\
I_{R} & = \pm \frac{2 e}{h} \int d \omega \int \frac{d \omega^{\prime}}{2 \pi Q} f_{R}(\omega) D^{<}\left(\omega^{\prime}\right) \operatorname{Tr}\left[\boldsymbol{\Gamma}^{\mathbf{R}} \mathbf{G}^{>}\left(\omega^{\prime}+\omega\right)\right] \\
& +\left(1-f_{R}(\omega)\right) D^{>}\left(\omega^{\prime}\right) \operatorname{Tr}\left[\boldsymbol{\Gamma}^{\mathbf{R}} \mathbf{G}^{<}\left(\omega^{\prime}+\omega\right)\right] . \quad \text { (A4) }
\end{aligned}
$$

Using Eqs. (10) for the boson self-energies, and some algebra, the difference between both expressions becomes

$I_{L}-I_{R}=\frac{-e}{h} \int \frac{d \omega^{\prime}}{Q}\left[D^{<}\left(\omega^{\prime}\right) \Pi^{>}\left(\omega^{\prime}\right)-D^{>}\left(\omega^{\prime}\right) \Pi^{<}\left(\omega^{\prime}\right)\right]$,

which is easily seen to vanish using $D \lessgtr=D^{r} \Pi \lessgtr D^{a}$ [see Eqs. (14)].

\section{Appendix B: Numerical procedure}

In this appendix we give some details about the numerical solution of the system of integral equations. 
We first solve the system that determines the retarded and greater Green functions and self energies for the auxiliary particles, Eqs. (10) to (14). After the system converges, we solve self-consistenlty Eqs. (8) and (14) for the lesser quantities.

Due to the gauge symmetry of the Hamiltonian in the representation of the auxiliary particles, $f_{i \sigma} \rightarrow$ $\exp \left(i \lambda_{0} t\right) f_{i \sigma}, b \rightarrow f_{i \sigma} \exp \left(i \lambda_{0} t\right) b$, the Lagrange multiplier $\lambda$ in Eq. (15) can be shifted by an arbitrary number $\lambda_{0} 39$. This is equivalent to a shift in the energies of the slave particles or of the frequency as $\omega \rightarrow \omega+\lambda_{0}$. Therefore, we can either set $Q=\left\langle b^{\dagger} b+\sum_{i \sigma} f_{i \sigma}^{\dagger} f_{i \sigma}\right\rangle=1$ and calculate $\lambda$ at each iteration or fix $\lambda$, calculate $Q$ and divide by $Q$ in the evaluation of physical properties 38,39 .

In the present problem, the spectral functions of the pseudo-fermions have two different peaks in contrast to the single one present in the ordinary Anderson model. We found that fixing $\lambda$ as the solution of the equation $\operatorname{Re} D(\omega, \lambda)=0$ at each iteration, the position of the lowest peak of the auxiliary fermion spectral densities coincide with the zero of energy (the Fermi level) With this chice of $\lambda$, in order to make the numerical evaluations efficiently, we use a non equidistant frequency mesh $\omega_{i}$ with two different regions logarithmically spaced. One of them is aimed at the zero of energy $\omega=0$ and the other one is centered near to the position of the second peak of the fermion spectral functions, $\omega=\delta$. Finally, to generate the non equidistant mesh we follow reference 39 .
1 Nitzan A and Ratner M A, 2003 Science 3001384

2 Venkataraman L, Klare J E, Nuckolls C, Hybertsen M S and Steigerwald M L, 2006 Nature (London) 442904

${ }^{3}$ Galperin M, Ratner M A, Nitzan A and Troisi A, 2008 Science $\mathbf{3 1 9} 1056$

4 van der Molen S J and Liljeroth P, 2010 J. Phys. Condens. Matter 22133001

${ }^{5}$ Cuevas J C and Scheer E, 2010 Molecular Electronics: An Introduction to Theory and Experiment (World Scientific, Singapore)

6 Park J, Pasupathy A N, Goldsmith J I, Chang C, Yaish Y, Petta J R, Rinkoski M, Sethna J P, Abruña H D, P L McEuen and Ralph D C, 2002 Nature (London) 417722

7 Lian W, Shores M P, Bockrath M, Long J R and Park H, Nature (London) 2002417725

8 Roch N, Florens S, Bouchiat V, Wernsdorfer W and Balestro F, 2008 Nature (London) $\mathbf{4 5 3} 633$

9 Parks J J, Champagne A R, Costi T A, Shum W W, Pasupathy A N, Neuscamman E, Flores-Torres S, Cornaglia P S, Aligia A A, Balseiro C A, Chan G K-L, Abruña H D, and Ralph D C, 2010 Science 3281370

10 Florens S, Freyn A, Roch N, Wernsdorfer W, Balestro F, Roura-Bas P and Aligia A A 2011 J. Phys.: Condens. Matter 23243202

11 Cornaglia P S, Roura-Bas P, Aligia A A and Balseiro C A 2011 Europhys. Lett. 9347005

12 Roura-Bas P and Aligia A A 2009 Phys. Rev. B 80035308

13 Roura-Bas P and Aligia A A 2010 J. Phys.: Condens. Matter 22025602

14 Reed M A, Zhou C, Muller C J, Burgin T P and Tour J M, Science 278252 (1997).

15 Danilov A V, Kubatkin S, Kafanov S, Hedegård P, StuhrHansen N, Moth-Poulsen K, and Bj申rnholm T, 2008 Nano Lett. 81

16 Dadosh T, Gordin Y, Krahne R, Khivrich I, Mahalu D, Frydman V, Sperling J, Yacoby A, and Bar-Joseph I, 2005 Nature (London) 436677

17 Hettler M H, Wenzel W, Wegewijs M R, and Schoeller H, 2003 Phys. Rev. Lett. 90076805

18 Cardamone D, Stafford C A, and Mazumdar S, 2006 Nano Lett. 62422

19 Ke S-H, Yang W, and Baranger H U, 2008 Nano Lett. 8 3257
20 Begemann G, Darau D, Donarini A, and Grifoni M, 2008 Phys. Rev. B 77 201406(R)

21 Rincón J, Hallberg K, Aligia A A and Ramasesha S, 2009 Phys. Rev. Lett. 103, 266807

22 Pariser R and Parr R, 1953 J. Chem. Phys. 21 466; Pople J A, 1953 Trans. Faraday Soc. 491375.

23 Büttiker M, 1986 Phys. Rev. Lett. 571761.

24 Jagla E A and Balseiro C A, 1993 Phys. Rev. Lett. 70639

25 Hallberg K, Aligia A A, Kampf A P and Normand B, 2004 Phys. Rev. Lett. 93067203

26 Aligia A A, Hallberg K, Normand B and Kampf A P, 2004 Phys. Rev. Lett. 93076801

27 Friederich S and Meden V, 2008 Phys. Rev. B 77195122

${ }^{28}$ Lobos A M and Aligia A A, 2008 Phys. Rev. Lett. 100 016803; 2009 Physica B 404, 3306

29 Rincón J, Aligia A A, and Hallberg K 2009 Phys. Rev. B 79035112

30 Roura-Bas P, Tosi L, Aligia A A, and Hallberg K, 2011 Phys. Rev. B 84, 073406

31 Izumida W, Sakai O, and Shimizu Y, 1998 J. Phys. Soc. Jpn. 67, 2444

32 Meden V and Marquardt F, 2006 Phys. Rev. Lett. 96 146801

33 Bohr D and Schmitteckert P, 2012 Ann. Phys. 524, 199

${ }^{34}$ Nilsson H A, Karlström O, Larsson M, Caroff P, Pedersen $\mathrm{J}$ N, Samuelson L, Wacker A, Wernersson L-E and Xu H Q, 2010 Phys. Rev. Lett. 104186804

35 Tosi L and Aligia A A, 2011 Phys. Status Solidi B 248732

36 Hatano T, Kubo T, Tokura Y, Amaha S, Teraoka S, and Tarucha S, 2011 Phys. Rev. Lett. 106, 076801

37 Lobos A M and Aligia A A, 2006 Phys. Rev. B 74165417

38 Wingreen N S and Meir Y, 1994 Phys. Rev. B 4911040

39 Hettler M H, Kroha J and Hershfield S, 1998 Phys. Rev. B 585649

40 Costi T A, Kroha J and Wölfle P, 1996 Phys. Rev. B 53 1850

41 Roura-Bas P, 2010 Phys. Rev. B 81, 155327

42 Oguri A, 2005 J. Phys. Soc. Jpn. 74110

43 Aligia A A, 2012 J. Phys. Condens. Matter 24015306 ; references therein.

44 Vaugier L, Aligia A A and Lobos A M, 2007 Phys. Rev. B 76165112

45 Freyn A and Florens S, 2011 Phys. Rev. Lett. 107017201 
46 Tosi L, Roura-Bas P, and Aligia A A, 2012 Physica B 407 3259

47 Lim J S, Choi M-S, Choi M Y, López R and Aguado R, 2006 Phys. Rev. B $\mathbf{7 4} 205119$

48 Anders F B, Logan D E, Galpin M R, and Finkelstein G, 2008 Phys. Rev. Lett. 100086809

49 Lipinski S and Krychowski D, 2010 Phys. Rev. B 81115327

50 Büsser C A, Vernek E, Orellana P, Lara G A, Kim E H, Feiguin A E, Anda E V and Martins G B, 2011 Phys. Rev. B 83125404

51 Jarillo-Herrero P, Kong J, van der Zant H S J, Dekker C, Kouwenhoven L P and De Franceschi S, 2005 Nature (London) 434484

52 Makarovski A, Liu J, and Finkelstein G, 2007 Phys. Rev. Lett. 99066801

53 Tettamanzi G C, Verduijn J, Lansbergen G P, Blaauboer M, Calderón M J, Aguado R, and Rogge S, 2012 Phys. Rev. Lett. 108046803

54 Pustilnik M and Glazman L I, 2001 Phys. Rev. Lett. 87, 216601

55 Grove-Rasmussen K, Grap S, Paaske J, Flensberg K, Andergassen S, Meden V, Jorgensen H I, Muraki K, and Fujisawa T, 2012 Phys. Rev. Lett. 108, 176802

${ }^{56}$ Izumida W, Sakai O, and Suzuki S, 2001 J. Phys. Soc. Jpn. 701045

57 Costi T A, 2001 Phys. Rev. B 64 241310(R)

58 Aligia A A and Proetto C R 2002 Phys. Rev. B 65165305

${ }^{59}$ Costi T A and Zlatić V, 2010 Phys. Rev. B 81, 235127
60 Lifshitz E M and Pitaevskii A L, 1981 Physical Kinetics (Pergamon, Oxford)

61 Mahan G D, 2000 Many Particle Physics (Kluver/Plenum, New York)

62 In the general case, Eqs. (14) contain an additional term of the form $\left|G^{r} / g^{r}\right|^{2} g^{\lessgtr}$ (see for example Eq. (7) of Ref. ${ }^{63}$ ), but they vanish in this case because $\left(g_{i j, \sigma}^{r}\right)^{-1}=\delta_{i j}\left(\omega-\tilde{E}_{i}\right)$ and $g^{\lessgtr} \sim \delta_{i j} \delta\left(\omega-\tilde{E}_{i}\right)$.

63 Aligia A A, 2006 Phys. Rev. B 74155125

64 Meir Y and Wingreen N S 1992 Phys. Rev. Lett. 682512

65 Jauho A P, Wingreen N S, and Meir Y, 1994 Phys. Rev. B 50, 5528

${ }^{66}$ Logan D E, Eastwood M P and Tusch M A, 1998 J. Phys.: Condens. Matter 102673

67 Levy Yeyati A, Martin-Rodero A, and Flores F, 1993 Phys. Rev. Lett. 712991

68 Monreal R C, Flores F, and Martin-Rodero A, 2010 Phys. Rev. B 82235412

69 Tosi L, Roura-Bas P, Llois A M, and Aligia A A, 2012 Physica B 4073263

70 Varma C M and Yafet Y, 1976 Phys. Rev. B 132950

71 Langreth D C 1966 Phys. Rev. 150516

72 Härtle R and Thoss M, 2011 Phys. Rev. B 83115414

73 Cornaglia P S, Usaj G, and Balseiro C A, 2007 Phys. Rev. B $76241403(\mathrm{R})$

${ }^{74}$ Härtle R, Butzin M, Rubio-Pons O, and Thoss M, 2011 Phys. Rev. Lett. 107046802

75 Kaasbjerg K and Flensberg K, 2011 Phys. Rev. B 84 115457 